\title{
Raising awareness of the urological manifestations of multiple sclerosis
}

\author{
James W.L. Wilson, MD, FRCSC, FACS
}

Department of Urology, Queen's University, Kingston, ON, Canada

Cite as: Can Urol Assoc J 2017;11 (1-2):65. http://dx.doi.org/10.5489/cuaj.4389

See related article on page 61 .

M ultiple sclerosis (MS) is a degenerative neurological disease of unknown etiology characterized by demyelination of central nervous system (CNS) axons and the presence of plaques. Considering that the neural pathways that govern micturition are as long as any in the CNS, it is not surprising that voiding function is frequently affected by MS. The plaques affect transmission of neural signalling so that although voiding symptoms are an infrequent initial presentation of MS, lower urinary tract symptoms (LUTS) are common over the course of the disease process.

As Canada has one of the highest rates of MS in the world, Canadian urologists need to be knowledgeable about MS, its natural history, and the therapeutic options. Despite significant advances in the treatment options for the various forms of MS, voiding function is rarely ameliorated by the primary MS treatment regimen. Patients present to urologists with an almost complete range of LUTS - irritative and obstructive, along with an increased risk of the usual complications associated with altered voiding (urinary infection, bladder and renal lithiasis). Aharony et al contribute to the literature on voiding dysfunction associated with MS in the current review and provide an update on the management of these patients.

What has not changed over the years is that investigation and management has to be tailored to each individual patient and that these patients need to be followed, as the symptoms may change during the course of disease. Treatment is rarely consistently effective over time and a change of therapy may be necessary. Urodynamics will guide therapy and is an important investigation initially, since symptoms frequently do not correlate with urodynamic findings. Blaivas et al reported many years ago that up to $73 \%$ of patients were treated inappropriately when urodynamics were not done. ${ }^{1}$ More than 35 years ago, Awad et al reported on medical therapy for MS-related voiding symptoms. ${ }^{2}$ The therapeutic options remain limited. Certainly, the available options for detrusor overactivity have advanced with the newer anticholinergics, beta-3 agonists, and onabotulinum

toxin. However, the treatment options for detrusor sphincter dyssynergia are very limited and other than the option of detrusor paralysis with onabotulinum toxin and intermittent catheterization, there have been no significant advances in the last 30 years. The chronically catheterized bladder, although occasionally the only viable option in severely disabled patients, really has to be regarded as a urological treatment failure.

What Aharony et al report that has changed is the need for investigation with cystoscopy, urodynamics, and upper tract imaging in followup of stable patients. Upper tract deterioration is rare in this patient population and routine followup imaging is not recommended. Similarly, routine cystoscopy is not necessary unless indicated by alternative symptoms, such as hematuria, suspicion of stones, etc. Urodynamics do not need to be repeated unless there is a change in symptoms or treatment failure. In an era of efficient use of healthcare resources, this is an important message.

Nonetheless, the goals of management remain the same: preservation of kidney function (avoidance of urinary infection, low pressure storage) and pragmatic approach to voiding symptom management, i.e., focussing on feasibility and patient acceptance while recognizing that complete resolution of urinary tract symptoms is a challenge that is frequently unrealized.

Hopefully, newer treatments will continue to emerge. In the meantime, urologists, need to be aware of the urological manifestations of MS and their treatment options.

Competing interests: The author reports no competing personal or financial interests.

\section{References}

1. Blaivas JG, Holland NJ, Giesser B, et al. Multiple sclerosis bladder: Studies and care. Ann NY Acad Sci 1984;436:328-45. https://doi.org/10.1111/i.1749-6632.1984.tb14804.x

2. Awad SA, Wilson JW, Fenemore J, et al. Dysfunction of the detrusor and urethra in multiple sclerosis: The role of drug therapy. Can J Surg 1982;25:259-62.

Correspondence: Dr. James W.L. Wilson, Department of Urology, Queen's University, Kingston, ON, Canada; wilsonil@KGH.KARI.NET 